\title{
Efecto de fibras micro y nanométricas de carbono en el comportamiento dinámico- mecánico y tribológico de la poliéter-éter cetona
}

\author{
Bladimir Ramón Valencia ${ }^{1} \mid$ Jose Ramon Sarasua $^{2}$ | Elena Fuentes ${ }^{3}$
}

Recibido:

Mayo 26 de 2011

Aceptado:

Diciembre 10 de 2011

\section{Resumen}

En este artículo, se analiza el efecto de la incorporación de fibras micrométricas de carbono (FC) y nanofibras de carbono (NFC), en una matriz termoplástica de poliéter-éter cetona (PEEK), sobre las propiedades dinámico-mecánicas y tribológicas de los compuestos obtenidos. Se elaboraron compuestos de PEEK reforzado con fibras de carbono micrométricas y nanométricas mediante la técnica de extruido y moldeado por inyección, en diferentes proporciones $(0,6 ; 1 ; 3$ y $5 \%$ en peso). Las probetas se enfriaron desde el estado fundido en un molde refrigerado con agua, obteniendo diferentes grados de cristalinidad. A fin de contrarrestar la disparidad de la cristalinidad, se realizó un tratamiento térmico a las diferentes formulaciones, con el objeto de homogenizar la estructura cristalina del PEEK. Las propiedades dinámico-mecánicas mostraron incrementos de la rigidez por debajo de la temperatura de transición vítrea para los sistemas que contenían refuerzo, siendo la concentración con mayor cantidad de fibras (micro/nano), las que presentaban mejor comportamiento con respecto a la matriz sin refuerzo. Así mismo, se realizó un estudio tribológico llevando a cabo ensayos oscilatorios, bajo movimiento lineal-recíproco de alta frecuencia, en un tribómetro SRV con los diferentes tipos de compuestos, evaluando su comportamiento en fricción y desgaste. En este sentido, la matriz termoplástica sin refuerzo ofreció el peor comportamiento en cuanto a fricción y desgaste, registrando el coeficiente de fricción más alto y la mayor profundidad de huella. La adición de diferentes porcentajes de fibra de carbono, implicó una reducción del coeficiente de fricción y del desgaste, tanto mayor cuanto más alta fue la proporción de fibra añadida.

ISSN 0122-820X

PP: $37-44$
${ }^{1}$ Grupo de Investigación en Ingeniería Mecánica de la Universidad de Pamplona (GIMUP), Departamento de Ingeniería Mecánica, Universidad de Pamplona, Colombia. hbladimir@ unipamplona.edu.co.

${ }^{2}$ Grupo de Investigación de Biopolímeros y Materiales Termoplásticos

(BMTP), Departamento de Minería y Metalúrgica y Ciencia de los Materiales, Universidad del

País Vasco, España. jr.sarasua@ ehu.es

${ }^{3}$ Dpto de Procesos de Fabricación, Fundación Tekniker, Eibar, España
Palabras clave: Compuestos, nanofibras de carbono, matriz termoplástica, propiedades dinámico-mecánicas y tribológicas.

\section{Abstract}

In this work, the effect of carbon nano-and microfibers on the dynamic mechanical and tribological properties of the thermoplastic polymer polyether ether ketone 
(PEEK) was studied. The compound materials were obtained by blending PEEK and carbon nano- and microfibers in different proportions $(0.6,1,3$ and $5 w t \%$ of fibers) using melt extrusion and injection molding. The samples were injected into a mold and cooled with water. Thereby different degrees of crystallinity of the polymer were obtained. Therefore, all samples were heat treated after the cooling process in order to homogenize the crystalline structure of PEEK. The characterization of the dynamic mechanical properties of the reinforced samples showed an increase in the stiffness below the glass transition temperature compared to pristine PEEK. The performance could be further improved by increasing the amount of carbon fibers where blends with $5 w t \%$ of fibers exhibited the highest stiffness values. Furthermore, tribological tests were conducted under oscillation at high frequency in reciprocal-linear motion in a SRV tribometer to determine the friction and wear coefficient of the samples. The pristine PEEK provided the worst performance revealing the highest friction and wear coefficient of all samples. The addition of carbon fibers reduced the friction and wear coefficient and the best results were obtained with high loads of carbon fibers.

Keywords: Composites, PEEK, carbon nanofibers, thermoplastic matrix, dynamic mechanical and tribological properties.

\section{Introducción}

$\mathrm{D}$ ebido a su elevada resistencia mecánica y sumada a su baja densidad, las fibras de carbono se han convertido en un material de refuerzo por excelencia para materiales poliméricos, incrementando las propiedades mecánicas de resinas poliméricas tanto termoestables como de tipo termoplástico [1].

Entre las aplicaciones más importantes de las fibras de carbono se pueden señalar sectores industriales, donde equipos y partes mecánicas deben poseer características de máxima exigencia mecánica y en donde el costo pueda justificar su funcionamiento. La industria militar, aeroespacial y la del deporte, tanto el de alta competición como el biomédico, son algunos ejemplos de sectores en donde se pueden incorporar piezas diseñadas para fabricación de accesorios en fibra de carbono [2].

Las nanofibras de carbono han despertado en los últimos años un gran interés en la comunidad científica, por sus excelentes propiedades mecánicas y eléctricas así como por sus buenas características térmicas, sobresaliendo su adecuada conductividad térmica [3].

Las NFC se obtienen a partir de la descomposición térmica de hidrocarburos, tales como el benceno y el metano, en presencia de partículas metálicas que actúan como agentes catalizadores a través de un lecho fluidizado [4]. Actualmente se registran en la literatura varios trabajos de investigación de compuestos de matriz PEEK empleando como refuerzo nanofibras de carbono [5,6].

Varios autores han reportado sus resultados en numerosas publicaciones, explicando la influencia de las nanofibras de carbono en las propiedades visco-elásticas del PEEK, entre los más representativos se tiene a J. Sandler y su grupo de colaboradores [5] con resultados 
similares a los obtenidos en este trabajo, principalmente con incrementos del módulo de almacenamiento $G$ ' con cantidades del $15 \%$ en peso de NFC.

Por otra parte, los aportes en la literatura, relacionados con las propiedades tribológicas de polímeros, es escasa, probablemente se debe a su estructura bifásica y al comportamiento visco-elástico. Si ahora se incorpora otro componente como la fibra de carbono, su interpretación relacionada con la fricción y el desgaste sería aun más compleja. Por tanto, en el presente trabajo, se analizan los parámetros que permitan explicar los fenómenos que conllevan la incorporación de fibras de tamaño micrométrico y nanométrico de carbono en las propiedades dinámico-mecánicas y tribológicas de la poliéter éter cetona.

\section{Materiales y métodos}

Lasnanofibrasdecarbono (NFC), empleadas en este estudio, fueron suministradas por el Grupo Español Antolín S.A. Las NFC están constituidas por redes hexagonales de átomos de carbono, enrollados en espiral, formando cilindros concéntricos. Su forma presenta la típica estructura conocida como de bambú [7], con diámetros de fibra que oscilan entre los 40-400 nm y longitudes que superan las 80 $\mu \mathrm{m}$. Poseen excelentes propiedades mecánicas con valores de módulo de Young alrededor de los 200 GPa [3]. La matriz de poliéter-éter cetona (PEEK) utilizada fue grado Victrex 450G, suministrada en forma de granza. Este polímero aromático lineal es semicristalino, con una temperatura de transición vítrea $(\mathrm{Tg})$ de $143^{\circ} \mathrm{C}$ y una temperatura de fusión $(\mathrm{Tm})$ de $343^{\circ} \mathrm{C}$. Dentro de los materiales de partida se utilizó PEEK/30\% FC de grado 450CA30 que, mediante su mezcla con PEEK puro, dio lugar a los compuestos PEEK/FC.

Los compuestos de PEEK reforzado con fibras/nanofibras de carbono se prepararon en una extrusora Brabender Plasticolder PL 2000. Se elaboraron compuestos de PEEK reforzado con fibras de carbono micrométricas y nanométricas en diferentes proporciones $(0,6 ; 1 ; 3$ y $5 \%$ en peso). La preparación de los compuestos se llevó a cabo en una máquina Inyectora Meteor 100/30 H a 380 ${ }^{\circ} \mathrm{C}$. Las muestras se enfriaron en el molde utilizando agua como medio de refrigeración. Posteriormente, a fin de garantizar el mismo grado de cristalinidad en todas las muestras, se llevó a cabo un tratamiento térmico de recocido a $220^{\circ} \mathrm{C}$ durante 30 minutos.

El estudio dinámico-mecánico se llevó a cabo en un Analizador Dinámico-Mecánico Mettler-Toledo DMA/SDTA861, a una velocidad de calentamiento de $3{ }^{\circ} \mathrm{C} / \mathrm{min}$ y una frecuencia de $1 \mathrm{~Hz}$, empleando un sistema de mordazas en modo de cizalla.

Los ensayos tribológicos de fricción y desgaste se llevaron a cabo en el Tribómetro SRV III de Optimol, que ofrece un movimiento oscilatorio lineal-recíproco de alta frecuencia, siguiendo una configuración de "bola sobre disco", de acuerdo a la norma ASTM D6425. Las condiciones fueron: carga de 50/150N, frecuencia oscilación de $10 \mathrm{~Hz}$, amplitud oscilación de $2 \mathrm{~mm}$, temperatura de $25^{\circ} \mathrm{C}$ y 30 minutos de duración. Se emplearon bolas del tradicional acero de rodamientos AISI 52100 (C: $1 \%, M n: 0,35 \%$, Si: 0,3\%, Cr: $1,5 \%, \mathrm{P}<0,025 \%, \mathrm{~S}<0,025 \%$ en peso) de $10 \mathrm{~mm}$ de diámetro, dureza: $60 \pm 2 \mathrm{HRc}$ y rugosidad, Ra: 0,025 $\pm 0,005 \mu \mathrm{m}$. Los discos de material polimérico empleados tenían 20 mm de diámetro

\section{Resultados y discusión}

\section{Análisis dinámico-mecánico (DMA)}

ISSN 0122-820X 
con 5\% FC, que corresponde al $64 \%$ con respecto a la resina sin fibra. Estos valores son representativos, en el rango de temperaturas, entre $15^{\circ} \mathrm{C}$ y la temperatura de transición vítrea $140^{\circ} \mathrm{C}$. El comportamiento de la $\tan \delta$, que corresponde a la variación de la fase amorfa móvil, no presenta cambios significativos con la adición de fibras de carbono.

Figura 1. Análisis dinámico-mecánico de compuestos de PEEK/FC en función de la temperatura.

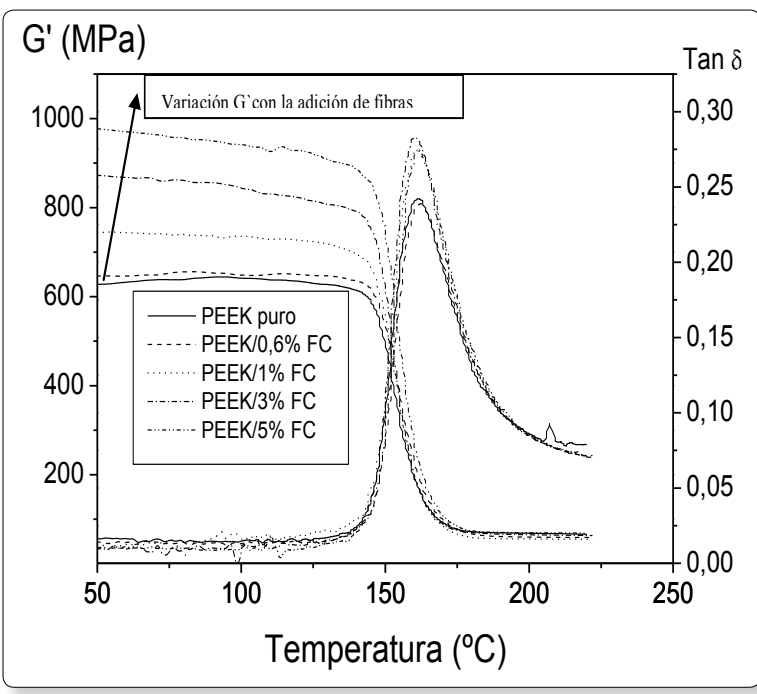

Para el sistema reforzado con nanofibras de carbono Figura 2, el comportamiento de las propiedades visco-elásticas es similar al encontrado con las fibras cortas de carbono. El módulo de almacenamiento (G'), se incrementa en un $50 \%$ para los compuestos con cantidades de NFC de 5\% en peso. Resultados similares se han reportado, en relación al incremento del módulo de almacenamiento del PEEK mediante la incorporación de nanotubos de carbono [8].

Otro aspecto a valorar es el incremento en la estabilidad térmica de la matriz, principalmente para contenidos en NFC del $5 \%$. Esto se atribuye, a la restricción del movimiento de las cadenas poliméricas, ejercidaporlas nanofibrasy comoconsecuencia se observa un ligero desplazamiento de la $\mathrm{Tg}$ hacia temperaturas mayores.
Figura 2. Análisis dinámico-mecánico de compuestos de PEEK/NFC en función de la temperatura.

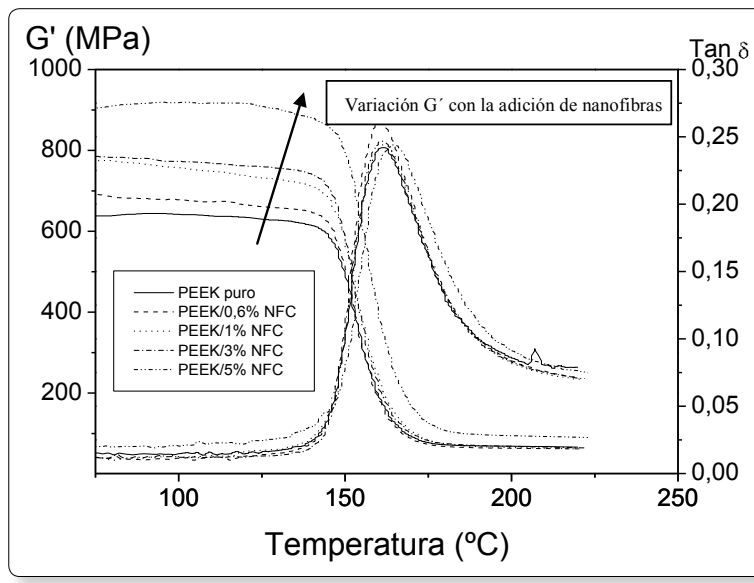

\section{Ensayos tribológicos de fricción y desgaste}

En la figura 3, se puede visualizar la evolución del coeficiente de fricción $(\mu)$, en el tiempo para los sistemas PEEK y PEEK/FC ensayados ante un disco de acero AISI 52100 usado para fabricar rodamientos.

Figura 3. Evolución del $\mu$ en el tiempo para PEEK y $\mathrm{PEEK} / \mathrm{FC}$ frente a acero rodamientos

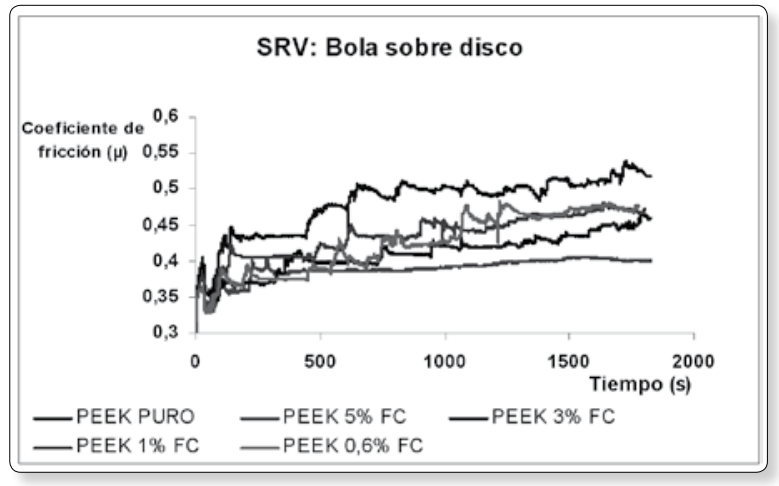

El mayor coeficiente de fricción lo registra la matriz termoplástica pura. La adición de diferentes porcentajes de fibra de carbono implica una disminución de dicho parámetro con respecto al de la matriz sin refuerzo. Destaca la formulación de PEEK/5\% FC, quien registró el mejor comportamiento en cuanto a fricción, presentando el coeficiente de fricción más bajo y estable. A medida que aumenta el porcentaje de fibra de carbono se detecta, en general, un menor coeficiente de 
Efecto de fibras micro y nanométricas de carbono en el comportamiento dinámico-

fricción, si bien, en el caso de los porcentajes más bajos, 0,6 y $1 \%$, se obtuvo un coeficiente de fricción estable similar entre ellos.

En cuanto al desgaste, únicamente se cuantificó la huella producida en los discos de material polimérico, empleando un microscopio confocal que permitió realizar topografías en $3 \mathrm{D}$ y perfiles en $2 \mathrm{D}$, siendo su resolución en la profundidad $Z$ de $10 \mathrm{~nm}$ y en el plano XY de 0,5 $\mu \mathrm{m}$.

En la figura 4 se visualiza que la máxima profundidad de huella (equivalente al mayor desgaste), se obtiene con la matriz PEEK pura. Al igual que ocurrió en fricción, el mejor comportamiento frente al desgaste lo ofrece el compuesto de PEEK/5\% FC (profundidad de huella entorno a $100 \mu \mathrm{m}$ ), con lo que se puede decir que con un $5 \%$ de fibras de carbono se reduce el desgaste en el disco en un 50\%. Las profundidades de huella obtenidas con un $0,6 \%, 1 \%$ y $3 \%$ en peso de fibra de carbono son muy similares entre sí $(150-130 \mu \mathrm{m})$, pero notablemente menores que la obtenida en el material PEEK puro $(200 \mu \mathrm{m})$.

Figuras 4. Perfiles en 2D de las huellas en los discos PEEK/\% FC

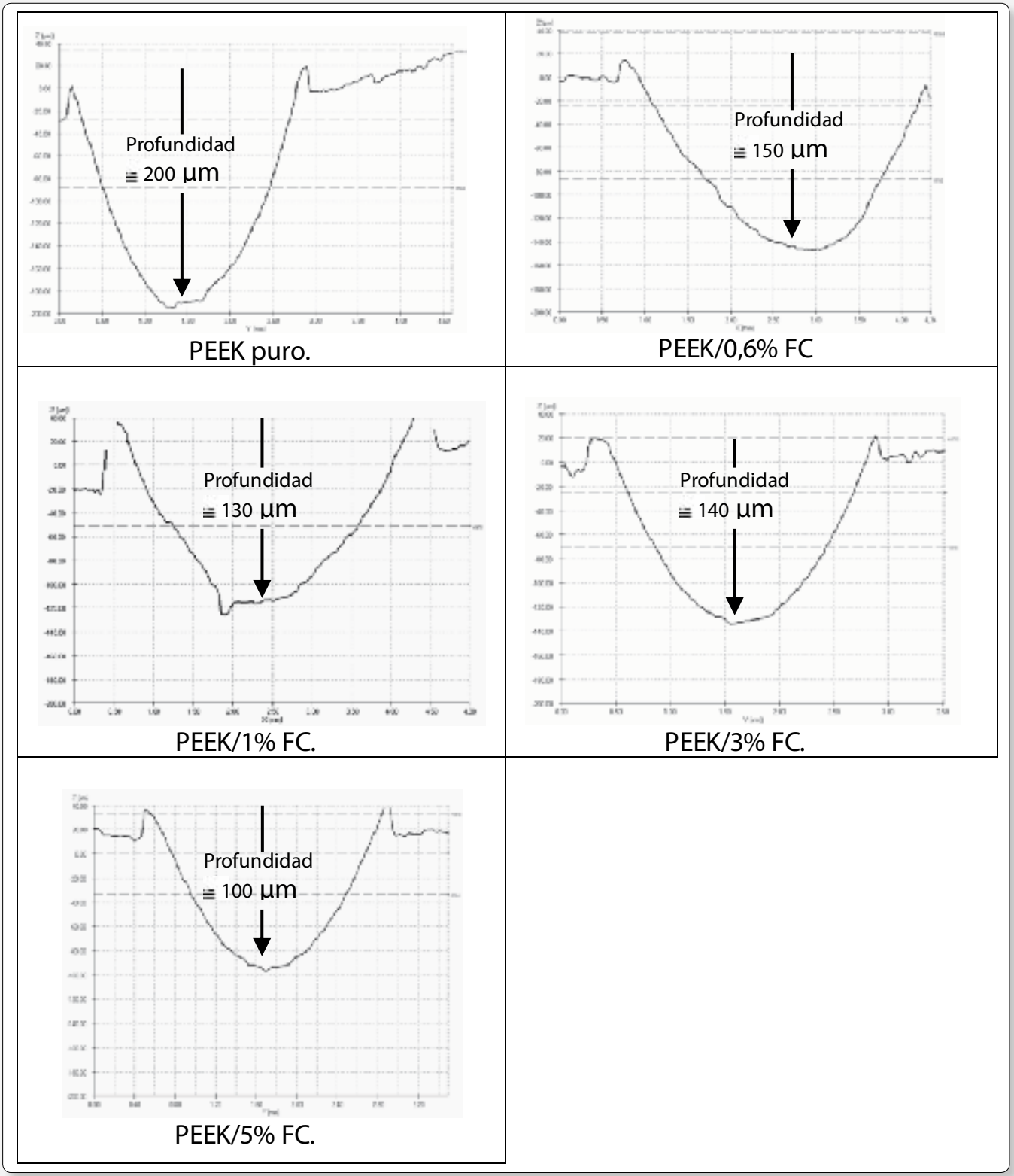


Las huellas en las bolas fueron analizadas mediante microscopía óptica. En el caso del material PEEK puro se consideran inapreciables. Sin embargo, las huellas obtenidas en las bolas de acero frente a los compuestos y nanocomposites se presentan bastante definidas, ofreciendo un aspecto de desgaste adhesivo (película de transferencia) más que de desgaste abrasivo.

En la figura 5, se visualiza la evolución del coeficiente de fricción en el tiempo para los sistemas PEEK y PEEK/NFC frente a un acero de rodamientos. mecánico y tribológico de la poliéter-éter cetona

Figura 5. Evolución del $\mu$ en el tiempo para PEEK y PEEK/NFC frente a acero rodamientos

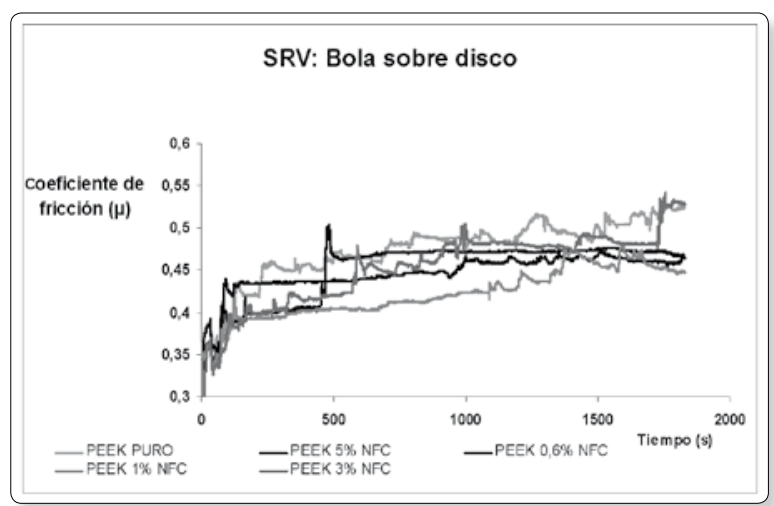

Figuras 6. Perfiles en 2D de las huellas en los discos PEEK/\% NFC

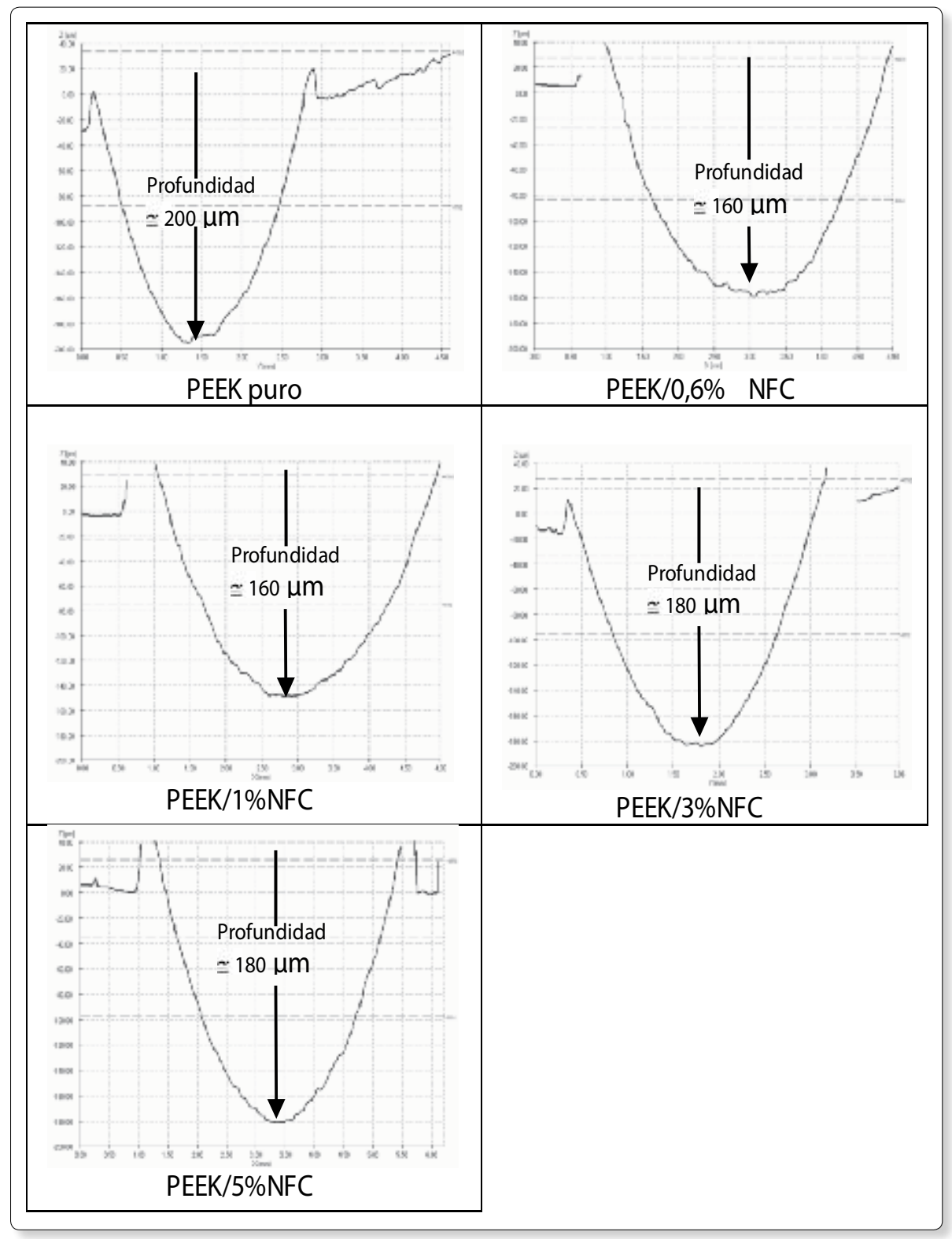


La adición de nanofibras de carbono reduce ligeramente el coeficiente de fricción. Si embargo, no se observa una tendencia en la reducción del coeficiente de fricción al adicionar nanofibras de carbono, siendo la formulación con $1 \%$ en peso de NFC la que presentó el menor coeficiente de fricción.

En cuanto al desgaste, nuevamente fue medida la huella producida en los discos de material polimérico, empleando un microscopio confocal. En las figuras 6, se pueden observar los diferentes perfiles, en 2D de las huellas formadas sobre los discos.

Nuevamente, la máxima profundidad de huella (correspondiente al mayor desgaste) se obtiene en el material PEEK puro. Con los distintos porcentajes de nanofibras de carbono estudiados se alcanzaron profundidades de huellas muy similares entre sí y no muy diferentes de las del PEEK puro.

En la tabla 1 se recogen los valores de coeficiente de fricción medio y de profundidad de huella en los discos, obtenidos para todos los tribosistemas analizados en las condiciones ensayadas

Tabla 1. Valores medios de coeficiente de fricción y profundidad de huella en discos fabricados con los diferentes compuestos analizados

\begin{tabular}{|c|c|c|}
\hline Disco & $\begin{array}{c}\text { Coeficiente de fricción } \\
\text { medio }(\mu)\end{array}$ & $\begin{array}{c}\text { Profundidad huellas } \\
\text { discos }(\mu m)\end{array}$ \\
\hline \multirow{2}{*}{ PEEK PURO } & 0,48 & \multirow{2}{*}{$\sim 200$} \\
\hline & 0,47 & \\
\hline \multirow{2}{*}{ PEEK/ 5\% FC } & 0,40 & \multirow{2}{*}{$\sim 100$} \\
\hline & 0,39 & \\
\hline \multirow{2}{*}{$\mathrm{PEEK} / \mathbf{3} \% \mathrm{FC}$} & 0,42 & \multirow{2}{*}{$\sim 140$} \\
\hline & 0,41 & \\
\hline \multirow{2}{*}{ PEEK $/ 1 \%$ FC } & 0,42 & \multirow{2}{*}{$\sim 130$} \\
\hline & 0,43 & \\
\hline \multirow{2}{*}{ PEEK/0,6 \% FC } & 0,42 & \multirow{2}{*}{$\sim 150$} \\
\hline & 0,43 & \\
\hline \multirow{2}{*}{$\mathrm{PEEK} / \mathbf{5} \% \mathrm{NFC}$} & 0,45 & \multirow{2}{*}{$\sim 180$} \\
\hline & 0,45 & \\
\hline \multirow{2}{*}{ PEEK $/ 3 \%$ NFC } & 0,45 & \multirow{2}{*}{$\sim 180$} \\
\hline & 0,44 & \\
\hline \multirow{2}{*}{$\mathrm{PEEK} / \mathbf{1} \% \mathrm{NFC}$} & 0,42 & \multirow{2}{*}{$\sim 160$} \\
\hline & 0,43 & \\
\hline \multirow{2}{*}{ PEEK $/ 0,6 \%$ NFC } & 0,43 & \multirow{2}{*}{$\sim 160$} \\
\hline & 0,44 & \\
\hline
\end{tabular}

El material PEEK puro registró el peor comportamiento en cuanto a fricción y desgaste, ofreciendo el coeficiente de fricción más alto y la mayor profundidad de huella. La adición de diferentes porcentajes de fibra de carbono, implica una reducción del coeficiente de fricción y desgaste, tanto mayor cuanto más alto es la proporción de fibra añadida. El resultado obtenido con un $0,6 \%$ y un $1 \%$ de fibra de carbono es muy similar, no observándose diferencia de comportamiento en cuanto a fricción y desgaste. Esta mejora de comportamiento tribológico observado, tras la adición de fibra de carbono no se pone de manifiesto cuando al material PEEK puro se le añaden nanofibras de carbono. La adición de nanofibras de carbono reduce muy ligeramente el coeficiente de fricción y el desgaste. Quizás estos porcentajes estudiados no sean los más adecuados para obtener las mejoras tribológicas deseadas.

\section{Conclusiones}

Tanto las fibras como las nanofibras de carbono representan una buena alternativa para la fabricación de composites que mejoren las propiedades mecánicas y tribológicas de una matriz termoplástica de poliéter-éter cetona.

Los resultados del análisis dinámicomecánico en los compuestos reforzados con fibras micro y nanométricas de carbono mostraron incrementos del módulo de almacenamiento $\left(G^{\prime}\right)$.

Diciembre 2011 ISSN 0122-820X
La estabilidad térmica fue afectada en el sistema que contiene NFC, debido a que a su tamaño nanométrico restringe el movimiento de las cadenas poliméricas y aumentan los valores de Tg.

Los ensayos tribológicos realizados revelaron el efecto positivo de la adición de las fibras de carbono a la matriz de PEEK pura. Sin embargo en el caso de las nanofibras de carbono esta mejora no resultó tangible. Esto pudo deberse a una pobre dispersión de las nanofibras de carbono en la matriz termoplástica, sugiriendo la posibilidad de 
incrementar el porcentaje de nanofibra de carbono añadido y/o mejorar la dispersión de las mismas en la matriz termoplástica para optimizar el comportamiento en cuanto a fricción y desgaste.

\section{Agradecimientos}

A la Universidad de Pamplona (Colombia), la Universidad del País Vasco (España), al Departamento de Procesos de Fabricación, Fundación Tekniker, Eibar (España), por los aportes económicos.

\section{Referencias bibliográficas}

[1] Hudnut S. W. et al. Use of submicron diameter carbon filaments for reinforcement between continuous carbon fiber layers in a polymer-matrix composite. Carbon, 33 (1995) 1627-1631.

[2] Soutis C. Carbon fiber reinforced plastics in aircraft construction. Materials Science and Engineering: A, 412 (2005) 171-176.

[3] Paredes J. I. et al. Nanocomposites de caucho butadieno-acrilonitrilo (NBR) reforzado con nanofibras de carbono. Revista PlásticosModernos, 90 (2005) 567-573.
[6] Sandler J. et al. Carbon-nanofibrereinforced poly (ether ether ketone) fibres. Journal of Materials Science. 38 (2003) 2135-2141.

[7] Werner P. et al. Tribological behaviour of carbon-nanofibre-reinforced poly(ether ether ketone). Wear 257 (2004) 10061014.

[8] Díez-Pascual A. et al. Development and characterizacion of PEEK/carbón nanotube composites. Carbón 47 (2009) $3079-3090$.

[4] Paredes J. I. et al. Characterization of submicron vapor grown carbon fibers by scanning tunneling microscopy. Carbon, 39 (2001) 1575-1587.

[5] Sandler J. et al. Carbon-nanofibrereinforced poly(ether ether ketone) composites. Composites Part A: Applied Science and Manufacturing, 33 (2002) 1033-1039. 